\title{
A Heuristic Approach for the Design of UAV-Based Disaster Relief in Optical Metro Networks
}

\author{
Evelin Cardoso ${ }^{1}$, Carlos Natalino ${ }^{2}$, Rodrigo Alfaia ${ }^{1}$, Anderson Souto ${ }^{2}$, Jasmine Araújo ${ }^{2}$, \\ Carlos R. L. Francês ${ }^{1}$, Luca Chiaraviglio ${ }^{(3,4)}$, and Paolo Monti ${ }^{2}$ \\ 1) Federal University of Pará, Belém, Brazil \\ E-mail: \{evelinhelena, rdalfaia, andersonvfs, rfrances, jasmine\}@ufpa.br \\ 2) Electrical Engineering Department, Chalmers University of Technology, Gothenburg, Sweden \\ E-mail: \{carlos.natalino, mpaolo\}@ chalmers.se \\ 3) EE Department, University of Rome Tor Vergata, Rome, Italy \\ E-mail: luca.chiaraviglio@uniroma2.it \\ 4) Consorzio Nazionale Interunivesitario per le Telecomunicazioni (CNIT), Rome, Italy
}

\begin{abstract}
We propose a novel algorithm to dimension the backup elements in an optical metro network, by considering the adoption of Unmanned Aerial Vehicles (UAVs) and wireless interfaces to realize backup wireless links. Our key idea is to efficiently find the set of node pairs that have to be connected by means of multi-hop UAV-based wireless links, which are selected based on the simulation of multiple disaster events. Results, obtained over a set of meaningful scenarios, demonstrate that our solution can greatly reduce the total installation costs compared to a naive approach, which is instead solely tailored to the restoration of the disrupted links in a given disaster scenario.
\end{abstract}

Keywords: Network design, UAV, Optical metro networks, Disaster management

\section{INTRODUCTION}

Optical metro networks are susceptible to a multitude of disasters (e.g., earthquakes, flooding), which can affect a substantial part of their infrastructure. Even if undamaged parts of the network may still be functional after a disaster event, the full recovery of a damaged optical network may take several days or weeks, thus impacting the performance levels provided to users during the reparation time [1].

The deployment of backup infrastructure is the traditional approach to face the damages brought by disaster events. In the context of metro networks, backup optical equipment is installed in addition to the devices that are used to serve the users in normal conditions. Clearly, this solution tends to notably increase the total installation costs compared to the case in which no backup elements are adopted [2], [3].

Recently, the use of Unmanned Aerial Vehicles (UAVs) has received great attention in several applications, including radio communications and search-and-rescue operations after disaster events (see e.g., [4], [5], [6], [7]). In this work, we consider the deployment of UAVs to realize backup connectivity in optical metro networks. Compared to other solutions, which require e.g., the installation of fixed links and fixed devices, the adoption of UAVs introduces several advantages, including cost reduction, flexibility, and easy installation. As a result, by leveraging the UAV capabilities, it is possible to design reliable, resilient and quickly deployable emergency communications network for disaster relief.

The main contributions of this paper are two-fold. Firstly, we define an optical metro architecture where wireless interfaces and UAVs are used as backup devices. Secondly, we propose a novel heuristic to: $i$ ) select the subset of optical nodes that have to be connected by means of UAV-based wireless backup links, and $i i$ ) dimension each wireless link in terms of required number of wireless interfaces and UAVs.

Our results, obtained by considering a realistic metro network and different technologies to provide UAVbased wireless connectivity, show that the proposed approach is able to perform consistently better (in terms of total costs and number of installed devices) compared to a solution targeting solely the replacement of the disrupted optical links with wireless ones. In particular, we demonstrate that the savings are gained thanks to the wise selection of backup links done by our approach, which in turn triggers the reduction of the number of deployed UAVs and wireless interfaces.

\section{UAV-Based Optical Metro Architecture}

Fig. 1 reports the considered UAV-based optical metro architecture. More in detail, the wired topology includes optical nodes that are connected by means of optical links. When a wireless link has to be installed over a given pair of nodes, the node pair has to be equipped with Radio Optical Interfaces (ROIs) and Radio Interfaces (RIs), in order to establish the two endpoints of the wireless link. Since the distance between the nodes in a metro network is far to be negligible, the wireless connectivity is assured thanks to the exploitation of a sequence of UAVs Base Stations (UAV-BSs), which are equipped also with RIs and act as relay nodes for the two endpoints of the wireless link.

The design of a UAV-based disaster relief infrastructure, as defined by the architecture in Fig. 1, needs to take into account the following key aspects. First of all, the ROIs and RIs that are deployed at the optical nodes have to be installed and configured before the disasters. Therefore, the problem of selecting the subset of nodes 


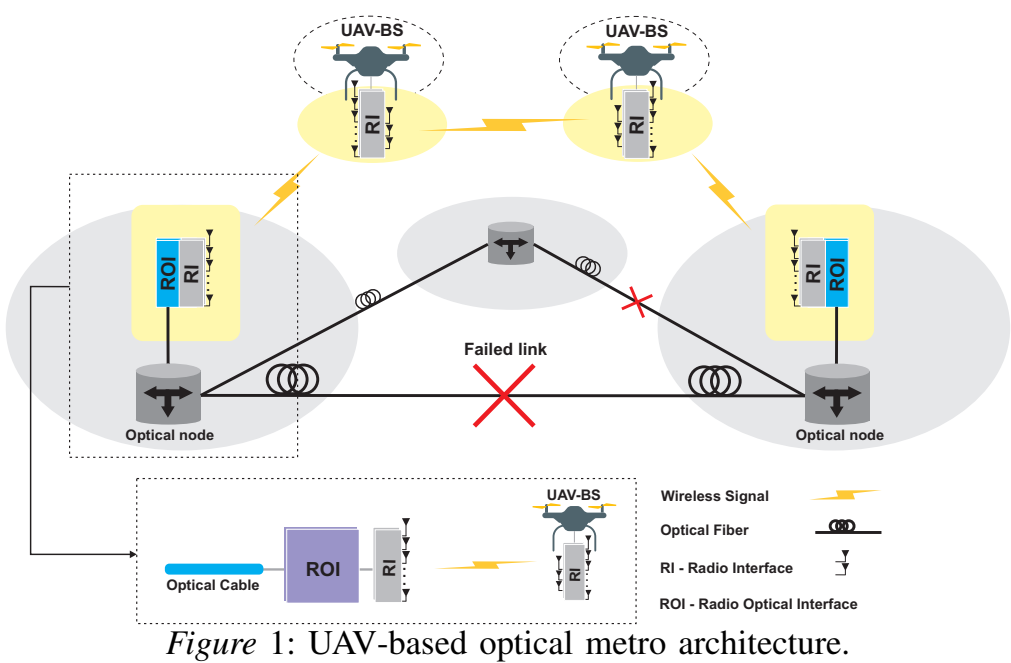

hosting ROIs and RIs is of crucial importance. Second, although the UAVs can be dynamically deployed after selecting the set of wireless links, the total number of such devices, as well as the RIs on board of them should be carefully take into account, by considering the amount of traffic that need to be sustained by each wireless link, as well as the maximum distance between RIs over the multi-hop wireless link.

In this scenario, an additional important aspect that has to be taken into account is how the routing is handled upon the occurrence of a disaster in a UAV-based optical metro architecture. By carefully routing the traffic over the optical links that have not been disrupted, in fact, it is possible to reduce both the number of wireless links and their amount of carried traffic, which is in turn beneficial to the reduction of the total costs for the considered UAV-based optical metro architecture.

\section{Algorithm Description}

In this section, we present the proposed heuristic, named UAV-based Disaster Relief Design (UAV-DRD). The objective of UAV-DRD is to minimize the total costs involved in deploying the infrastructure necessary for the disaster relief. For the sake of simplicity, the costs are expressed as a function of the total number of ROI, RI and UAVs.

More formally, we assume a metropolitan optical network denoted by a graph $G(\mathcal{N}, \mathcal{L})$ with $N=|\mathcal{N}|$ optical nodes and $L=|\mathcal{L}|$ optical links. Let us denote with $D_{(i, j)}$ the Euclidean distance between $i \in \mathcal{N}$ and $j \in \mathcal{N}$. Let $\Gamma_{(i, j)}$ be the traffic between nodes $i$ and $j$. Let $\mathcal{S}$ be the set of disaster scenarios after which the disaster relief infrastructure should be designed. Each disaster scenario $s \in \mathcal{S}$ contains a set of $\mathcal{L}_{s} \subset \mathcal{L}$ links that are disrupted by the disaster, i.e., upon disaster $s$, the network graph can be denoted as $G\left(\mathcal{N}, \mathcal{L} \backslash \mathcal{L}_{s}\right)$. The set $\mathcal{L}_{s}$ can be obtained in several ways, e.g., by solving a critical link set detection problem [8]. In general, the cardinality of $\mathcal{L}_{s}$ may range from few disrupted links that cause a degradation in the overall throughput that can be supported by the network, to massive failures that disconnect parts of the network forming different disconnected subgraphs.

Under normal conditions, the traffic in $\Gamma_{(i, j)}$ is routed over $G(\mathcal{N}, \mathcal{L})$. Upon the occurrence of disaster $s$, we assume that the network will first try to recover the traffic by exploiting the spare resources available in the resulting topology $G\left(\mathcal{N}, \mathcal{L} \backslash \mathcal{L}_{S}\right)$ in a process known as service restoration [9]. After this step, the remaining traffic that can not be served by the optical network, denoted by $\Theta_{(i, j)}$ for each node pair $(i, j)$, is served by new wireless links created with the help of UAVs. More in depth, let $\mathcal{T}$ be the set of available wireless technologies. Each wireless technology $t \in \mathcal{T}$ is characterized by a maximum distance $D_{t}^{\mathrm{MAX}}$ between two relay nodes and by a maximum capacity $C_{t}^{\mathrm{MAX}}$. Clearly, a given amount of traffic $\Theta_{(i, j)}$ can be routed through wireless link $(i, j)$ realized with technology $t$ only under the following two conditions: $i) \Theta_{(i, j)}<C_{t}^{\mathrm{MAX}}$ for each RI deployed over the link, and ii) maximum distance between deployed relays over the link lower than $D_{t}^{\mathrm{MAX}}$.

Alg. 1 illustrates the main steps of UAV-DRD. Intuitively, we design our solution by adhering to the following principles: (i) computation of the number of times that each node is selected to install RI and ROI interfaces to realize a wireless link, across all the disaster scenarios; (ii) computation of the backup infrastructure necessary to support each disaster individually, and (iii) computation of the total backup infrastructure over all the scenarios by analyzing the nodes with a given rule (based on (i) and (ii)). More formally, the UAV-DRD algorithm takes as input the network graph $G(\mathcal{N}, \mathcal{L})$, the set of disaster scenarios $\mathcal{S}$, the characteristics of wireless technology $t$, the traffic matrix $\Gamma$ and the matrix of node distances $D$. The solution then produces as output: the number of deployed ROIs $N^{\mathrm{ROI}}$, the number RIs to be carried by the UAVs $N^{\mathrm{RI}}$, and number of deployed UAVs $N^{\mathrm{UAV}}$.

In the first part (lines 1-7), UAV-DRD iterates over all the disaster scenarios, in order to compute how many times each node is used as endpoint of a backup link. In this step, we exploit the following intuitions: $i$ ) we select 


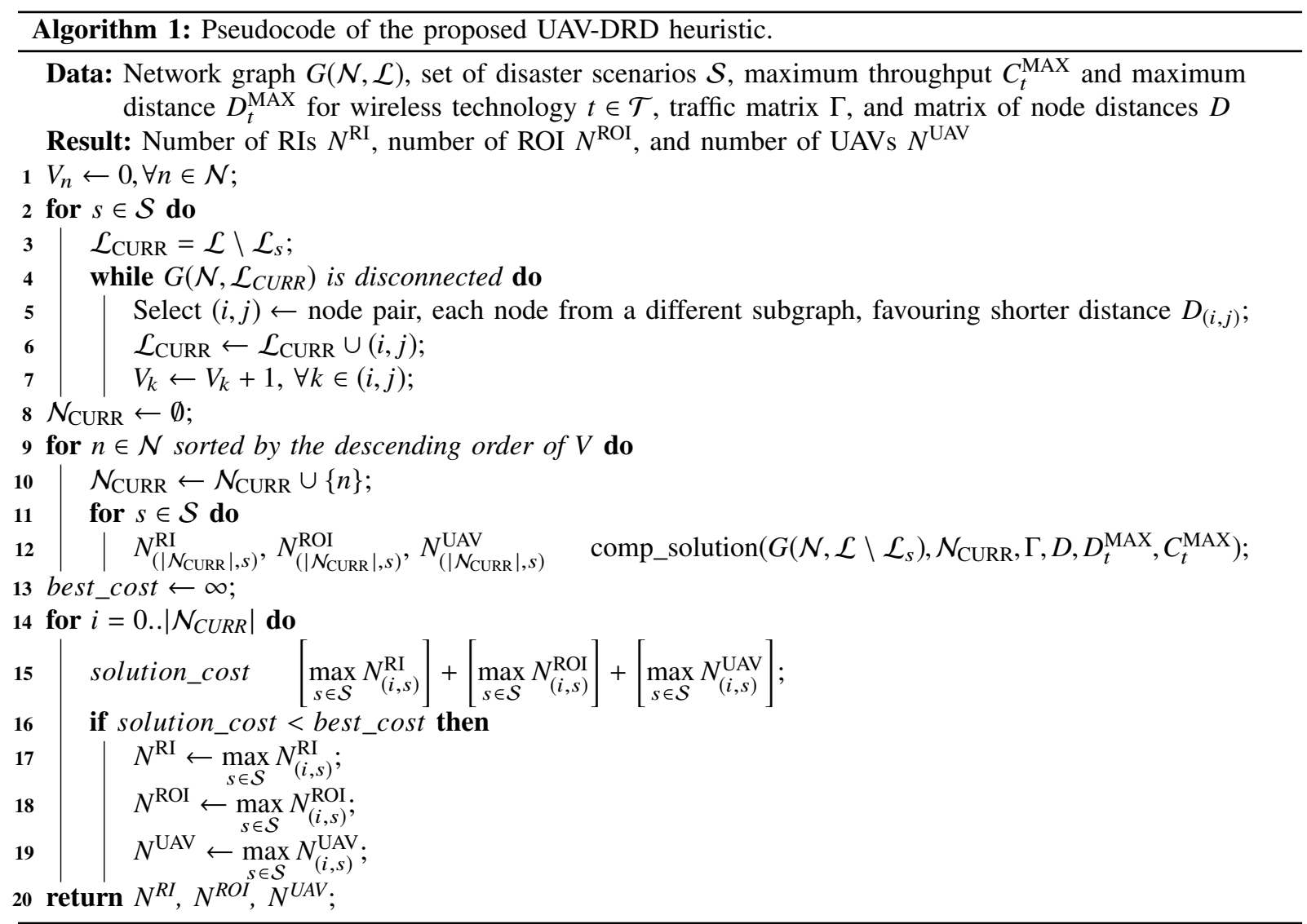

node pairs that enable the connection between two different subgraphs that were formed due to the disaster, prioritizing nodes with shorter distance $D_{(i, j)}$ (line 5) until the graph $G\left(\mathcal{N}, \mathcal{L}_{\mathrm{CURR}}\right)$ is connected again (line 4), and $i$ ) we increase the counter $V_{k}$ for each node $k$ appearing as endpoint of a wireless link $(i, j)$ (line 7).

In the following step (lines 8-12), the algorithm iterates over the set of nodes $\mathcal{N}$ and the set of scenarios $\mathcal{S}$, in order to derive the number of RIs, ROIs and UAVs for each disaster scenario. More in detail, the nodes in $\mathcal{N}$ are sequentially analyzed, starting from the ones that have the largest values of $V_{k}$ (line 9). The heuristic then computes the number of installed RIs, ROIs, and UAVs by considering the set of nodes with ROI and each scenario $s$ (line 12), taking into account: $i$ ) the current optical graph $G\left(\mathcal{N}, \mathcal{L} \backslash \mathcal{L}_{s}\right)$, ii) the current set of nodes under consideration $\mathcal{N}_{\mathrm{CURR}}$, the traffic matrix $\Gamma$, as well as the maximum capacity $C_{t}^{\mathrm{MAX}}$ and maximum distance $D_{t}^{\mathrm{MAX}}$. The comp_solution function generates a temporary graph including the working optical links as well as the wireless links formed by pairs of nodes with ROIs. Then, the traffic matrix is routed using the shortest path algorithm and allowing for traffic demands to be routed over multiple paths. We avoid using wireless links as much as possible by setting the weights of the links in the resulting topology. If the link between nodes $i$ and $j$ is optical, the link weight is equal to $D_{(i, j)}$. If the link is wireless, its weight is equal to $100 \times D_{(i, j)}$. Based on the amount of traffic routed over the wireless links, the heuristic computes and returns the number of installed RIs, ROIs, and UAVs associated with a number of nodes receiving ROIs (i.e., $\left|\mathcal{N}_{\mathrm{CURR}}\right|$ ) and a disaster scenario $s$ are stored (line 12). Finally, the heuristic finds the lowest solution cost with sufficient resources across all the disaster scenarios (lines 13-19).

\section{SCENARIOS UNDER CONSIDERATION}

In order to realize the wireless links, we consider a set of wireless technologies, namely IEEE 802.11 ay [10], Long-Term Evolution (LTE) [11], Free-Space Optical Communication (FSO) [12] and microwave (mW) [13]. To this aim, Table 1 reports the main features of each technology $t$ in terms of maximum distance $D_{t}^{\mathrm{MAX}}$ and maximum capacity $C_{t}^{\mathrm{MAX}}$. Interestingly, we can note that both the maximum distance and the maximum capacity notably vary across the set of technologies. For example, the capacity of IEEE 802.11ay is pretty large, but the distance is typically short (i.e., lower than $1 \mathrm{~km}$ ). On the other hand, the microwave technology can achieve large distances, but the rate is typically pretty low (i.e., lower than 2 Gbps).

Focusing then on the optical metro networks, Fig. 2 illustrates the adopted topology [14], which is based on Stockholm city (Sweden). The resulting graph encompasses $|\mathcal{N}|=33$ optical nodes and $|\mathcal{L}|=42$ optical links. Focusing on the traffic matrix $\Gamma$, we assume a random traffic matrix generated between the node pairs in the topology, with total traffic exchanged equal to $10 \mathrm{Tbps}$. 


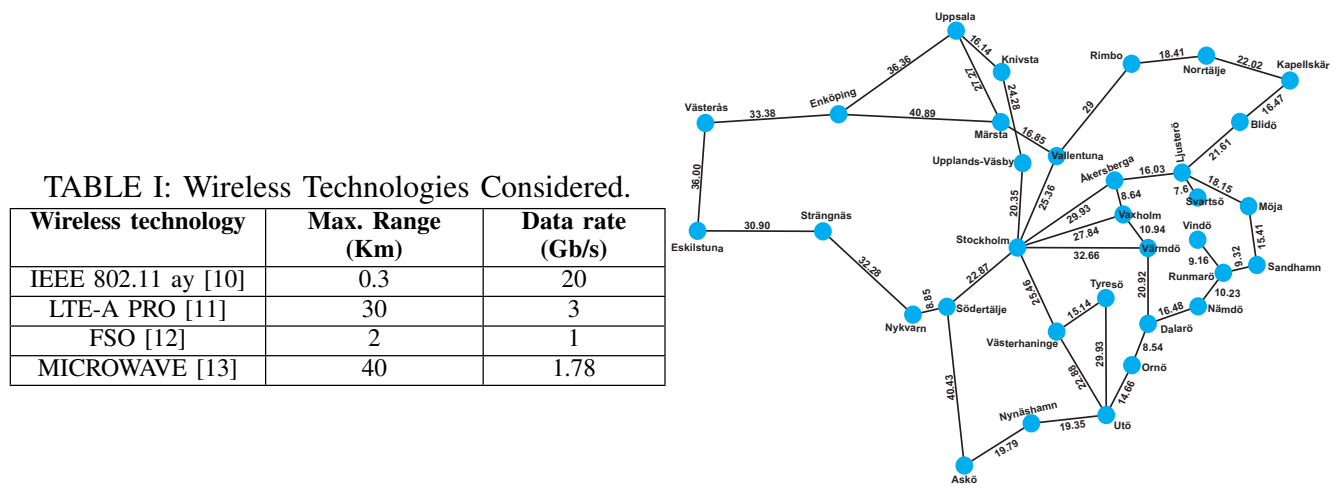

Figure 2: Topology.

Focusing on the disaster events, we use a traffic-aware version of the critical link set detection model described in [8] to compute the set of disrupted links $\mathcal{L}_{s}$ in each disaster scenario, based on the input topology and the generated traffic matrix. The number of links in $\mathcal{L}_{s}$ ranges between 2 and 12 , resulting in $|\mathcal{L}|=11$, thus representing between $4 \%$ and $28 \%$ of the total number of links in the topology, disrupted for the Stockholm topology and the traffic matrix considered. Finally, for the sake of simplicity, we assume a unitary (adimensional) cost for each RI, ROI and UAV.

\section{NUMERICAL RESULTS}

We then run UAV-DRD over the considered scenarios. In order to introduce a term of comparison, we also evaluate a naive heuristic, which greedily establish UAV-based wireless links only for disrupted links in each disaster scenario. Fig. 2 reports the impact of the percentage of recovered traffic vs. the following indicators: i) total (unitary) costs (Fig. 2a), ii) total number of RIs (Fig. 2b), total number of UAVs (Fig. 2c), and number of ROIs (Fig. 2d). The analysis of the figures reveals that all the considered metrics are increasing with the amount of recovered traffic (as expected). Interestingly, the total (unitary) costs are clearly reduced when the proposed UAV-DRD solution is employed, for all the considered wireless technologies. In addition, the lowest costs are achieved when the LTE technology is adopted. In particular, the total costs of the network designed

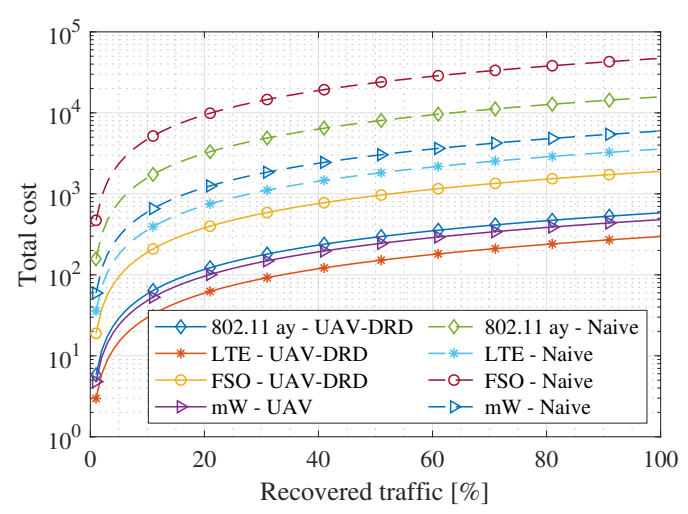

(a)

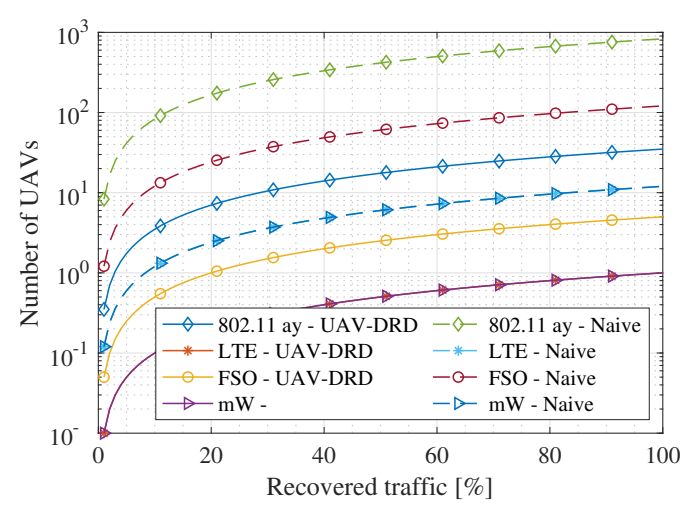

(c)

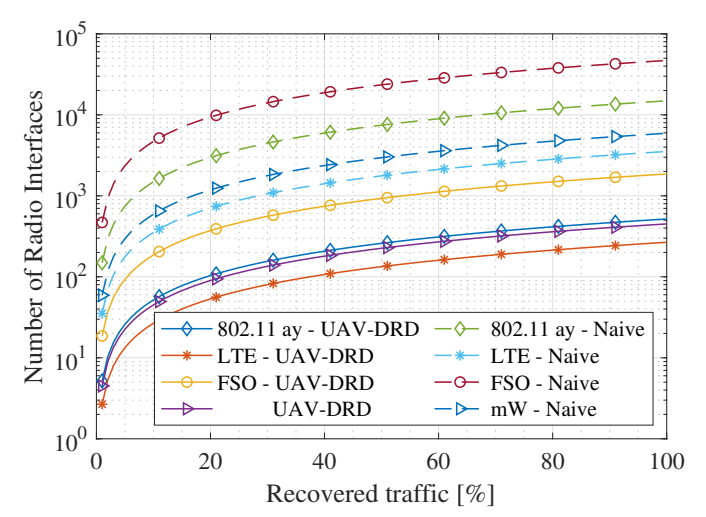

(b)

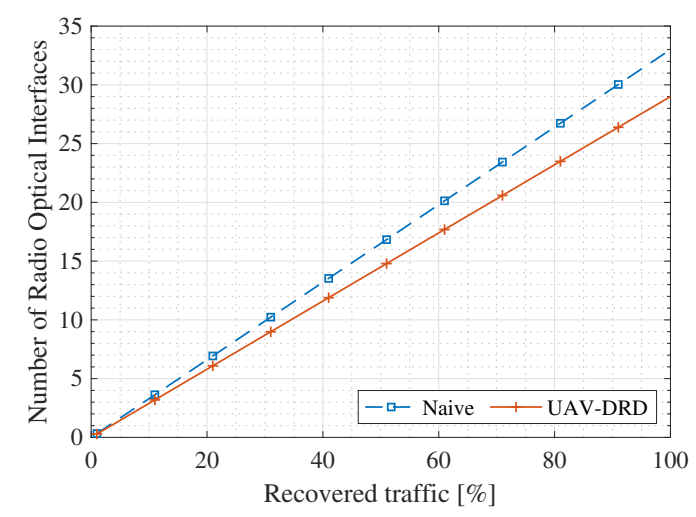

(d)

Figure 2: Comparison of the proposed UAV-DRD and the naive approach over the different metrics. 
by UAV-DRD with LTE is always around one order of magnitude lower than the one designed by the naive heuristic using the same technology (when the amount of recovered traffic is larger than 20\%).

In the following part, we move our attention to the number of deployed RIs and the number of deployed UAVs, shown in Fig. 2b and in Fig. 2c, respectively. We can see that the number of RIs is always clearly higher than the number of UAVs. Moreover, the different technologies strongly impact the obtained outcomes. Interestingly, both LTE and microwave require the same number of UAVs for both UAV-DRD and naive strategies, which is explained by a close trade-off between range and data rates supported by these technologies. In any case, however, the proposed UAV-DRD solution always performs better than the naive one.

Eventually, we evaluate the number of ROI interfaces, shown in Fig. 2d for the LTE case. Interestingly, this metric is also optimized by UAV-DRD, since our solution operates a wise choice when selecting the pairs of nodes that have to be connected through the UAV-based wireless links. On the other hand, the naive approach aims only at re-establishing solely the links which were disrupted by the disaster, leading to an increase of ROIs w.r.t. the UAV-DRD.

\section{Conclusions}

We have faced the restoration of the connectivity in an optical metro network subject to a disaster by proposing an architecture able to employ UAV-based wireless links. We have then designed UAV-DRD, a simple - yet effective - algorithm to compute the total number of RIs, ROIs and UAVs in a given optical topology. Results, obtained over a realistic metro network and a set of disaster scenarios, prove the efficacy of our solution w.r.t. a naive approach, which instead solely replaces the disrupted links with the wireless ones, without taking into account the impact on the total costs. On the other hand, we have shown that UAV-DRD always outperforms the naive solution. In particular, UAV-DRD is able to reduce the total costs by at least one order of magnitude w.r.t. the naive heuristic.

As future works, we plan the expand our work in the following areas: $i$ ) more detailed models to express the costs, ii) comparison of UAV-DRD w.r.t. optimal approaches, iii) evaluation of the proposed solution during the post-disaster phase, and $i v$ ) integration of multiple RIs on each UAV to realize multiple relays across multiple links (thus further redcing the costs).

\section{REFERENCES}

[1] S. Xu et al., "Field-trial of the recovery of fiber networks C/M-plane via an IoT-based narrow-band and lossy links system (FRENLL)," in OECC/PSC, 2019.

[2] M. Khalil et al., "Feasibility, architecture and cost considerations of using TVWS for rural internet access in 5G," in Conference on Innovations in Clouds, Internet and Networks (ICIN), 2017, pp. 23-30.

[3] C. Lange et al., "Access network economics: A total-cost-of-ownership perspective," in Optical Fiber Communications Conference and Exposition (OFC), 2018, p. Th1B.1.

[4] C. Luo et al., "Unmanned aerial vehicles for disaster management," in Geological Disaster Monitoring Based on Sensor Networks. Springer, 2019, pp. 83-107.

[5] K. G. Panda et al., "Design and deployment of UAV-aided post-disaster emergency network," IEEE Access, vol. 7, pp. 102 985-102999, 2019.

[6] L. Amorosi et al., "Optimal energy management of UAV-based cellular networks powered by solar panels and batteries: Formulation and solutions," IEEE Access, vol. 7, pp. 53698-53717, 2019.

[7] Z. Wang et al., "Adaptive deployment for UAV-aided communication networks," IEEE Trans. Wireless Commun., vol. 18, no. 9, pp. 4531-4543, 2019.

[8] A. de Sousa and D. Santos, "Vulnerability evaluation of networks to multiple failures based on critical nodes and links," in Guide to Disaster-Resilient Communication Networks, J. Rak and D. Hutchison, Eds. Springer, 2020, ch. 3.

[9] J. Ahmed et al., "Hybrid survivability schemes achieving high connection availability with a reduced amount of backup resources," IEEE J. Opt. Commun. Netw., vol. 5, no. 10, pp. A152-A161, 2013.

[10] D.-J. Deng et al., "On quality-of-service provisioning in IEEE 802.11 ax WLANs," IEEE Access, vol. 4, pp. 6086-6104, 2016.

[11] H. Ullah et al., "5G communication: An overview of vehicle-to-everything, drones, and healthcare usecases," IEEE Access, vol. 7, pp. 37 251-37 268, 2019.

[12] W. Fawaz et al., "UAV-aided cooperation for FSO communication systems," IEEE Communications Magazine, vol. 56, no. 1, pp. 70-75, 2018.

[13] CableFree. (2018, December) Cfmw-for3. [Online]. Available: https://www.cablefree.net/ cablefree-microwave/for3/

[14] M. Hadi and E. Agrell, "Joint power-efficient traffic shaping and service provisioning for metro elastic optical networks," IEEE J. Opt. Commun. Netw., vol. 11, no. 12, pp. 578-587, 2019. 